\title{
The existence of positive solutions for multi-point boundary value problem at resonance on the half-line
}

Weihua Jiang ${ }^{*}$ and Caixia Yang

\section{${ }^{*}$ Correspondence:}

weihuajiang@hebust.edu.cn

College of Sciences, Hebei

University of Science and

Technology, Shijiazhuang, Hebei 050018, P.R. China

\section{照 Springer}

\begin{abstract}
We establish new results on the existence of positive solutions for the multi-point boundary value problem at resonance on the half-line. Our results are based on the Leggett-Williams norm-type theorem due to O'Regan and Zima, which requires appropriate Banach spaces and proper operators. An example is given to illustrate the main results.

MSC: $34 \mathrm{~B} 15$

Keywords: positive solutions; multi-point boundary value; resonance; Fredholm operator; half-line
\end{abstract}

\section{Introduction}

In this paper, we will discuss the existence of positive solutions for the multi-point boundary value problem

$$
\left\{\begin{array}{l}
u^{\prime \prime}(t)+f(t, u(t))=0, \quad t \in[0,+\infty) \\
u(0)=0, \quad u^{\prime}(+\infty)=\sum_{i=1}^{m-1} \alpha_{i} u^{\prime}\left(\xi_{i}\right)
\end{array}\right.
$$

where $f \in C([0,+\infty) \times \mathbf{R} \rightarrow \mathbf{R}), f(t, 0)$ is not always equal to $0, t \in[0,+\infty), \alpha_{i}>0$, $\sum_{i=1}^{m-1} \alpha_{i}=1, i=1,2, \ldots, m-1,0=\xi_{1}<\cdots<\xi_{m-1}<\infty$.

Boundary value problems of differential equations are applied to more and more disciplines, and the existence of one or multiple positive solutions for multi-point BVPs has been attracting more and more authors, for details see [1-16]. Generally speaking, the boundary value problems of differential equations can be roughly divided into two parts. One is boundary value problems on the finite interval; Infante and Zima [17] obtained the existence of positive solutions for the problem

$$
\left\{\begin{array}{l}
x^{\prime \prime}(t)+f(t, x(t))=0, \quad t \in(0,1), \\
x^{\prime}(0)=0, \quad x(1)=\sum_{i=1}^{m-2} \alpha_{i} x\left(\eta_{i}\right),
\end{array}\right.
$$

with $0<\eta_{1}<\eta_{2}<\cdots<\eta_{m-2}<1, \alpha_{i}>0, \sum_{i=1}^{m-2} \alpha_{i}=1$. The other is boundary value problems on the infinite interval, for details see [18-22]; [20] obtained the existence of positive

(c) 2016 Jiang and Yang. This article is distributed under the terms of the Creative Commons Attribution 4.0 International License (http://creativecommons.org/licenses/by/4.0/), which permits unrestricted use, distribution, and reproduction in any medium, provided you give appropriate credit to the original author(s) and the source, provide a link to the Creative Commons license, and indicate if changes were made. 
solutions for the problem

$$
\left\{\begin{array}{l}
x^{\prime \prime}(t)=f\left(t, x(t), x^{\prime}(t)\right), \quad t \in(0,+\infty) \\
x(0)=x(\eta), \quad \lim _{t \rightarrow+\infty} x^{\prime}(t)=0
\end{array}\right.
$$

and

$$
\left\{\begin{array}{l}
x^{\prime \prime}(t)=f\left(t, x(t), x^{\prime}(t)\right)+e(t), \quad t \in(0,+\infty), \\
x(0)=x(\eta), \quad \lim _{t \rightarrow+\infty} x^{\prime}(t)=0,
\end{array}\right.
$$

where $f:[0,+\infty) \times \mathbf{R}^{2} \rightarrow \mathbf{R}, e:[0,+\infty) \rightarrow \mathbf{R}$ are continuous and $\eta \in(0,+\infty)$.

To the best of our knowledge, only few authors studied the existence of positive solutions for boundary value problems at resonance on the half-line. In [21], the authors dealt with the second order boundary value problem with integral boundary conditions on a half-line

$$
\left\{\begin{array}{l}
\left(p(t) x^{\prime}(t)\right)^{\prime}+g(t) f(t, x(t))=0, \quad \text { a.e. in }(0,+\infty) \\
x(0)=\int_{0}^{+\infty} x(s) g(s) d s, \quad \lim _{t \rightarrow+\infty} p(t) x^{\prime}(t)=p(0) x^{\prime}(0) .
\end{array}\right.
$$

In [22], the authors investigated the existence of positive solutions for the two-point problem at resonance on the half line,

$$
\left\{\begin{array}{l}
D_{0^{+}}^{\alpha} u(t)=f(t, u(t)), \quad t \in[0,+\infty), \\
u(0)=u^{\prime}(0)=u^{\prime \prime}(0)=0, \quad D_{0^{+}}^{\alpha-1} u(0)=\lim _{t \rightarrow+\infty} D_{0^{+}}^{\alpha-1} u(t),
\end{array}\right.
$$

where $D_{0^{+}}^{\alpha}$ is the standard Riemann-Liouville fractional derivative.

Inspired by the works above, we will study the existence of positive solutions for the problem (1.1).

Define 1.1 We call $u$ is a positive solution of the boundary value problem (1.1), if $u \geq 0$, $u \neq 0$, and satisfies the problem (1.1).

\section{Preliminaries}

Let us recall some standard facts and the Leggett-Williams norm-type theorem due to O’Regan and Zima.

Let $X, Y$ be real Banach spaces. A linear mapping $L: \operatorname{dom} L \subset X \rightarrow Y$ is called a Fredholm operator of index zero if $\operatorname{Im} L$ is closed and $\operatorname{dim} \operatorname{Ker} L=\operatorname{codim} \operatorname{Im} L<\infty$, which implies that there exist continuous projectors $P: X \rightarrow X$ and $Q: Y \rightarrow Y$ such that $\operatorname{Im} P=$ $\operatorname{Ker} L$ and $\operatorname{Ker} Q=\operatorname{Im} L$. Moreover, since $\operatorname{dim} \operatorname{Im} Q=\operatorname{codim} \operatorname{Im} L$, there exists an isomorphism $J: \operatorname{Im} Q \rightarrow \operatorname{Ker} L$. Denote by $L_{P}$ the restriction of $L$ to $\operatorname{Ker} P \cap \operatorname{dom} L \rightarrow \operatorname{Im} L$ and its inverse by $K_{P}$. So $K_{P}: \operatorname{Im} L \rightarrow \operatorname{Ker} P \cap \operatorname{dom} L$ and the coincidence equation $L x=N x$ is equivalent to $x=(P+J Q N) x+K_{P}(I-Q) N x$.

A nonempty convex closed set $C \subset X$ is said to be a cone provided that

(i) $\lambda x \in C$, for $x \in C, \lambda \geq 0$;

(ii) $x,-x \in C$ implies $x=0$. 
Note that every cone $C \subset X$ induces a partial order in $X$ by $x \preceq y$ if and only if $y-x \in C$. Let $\gamma: X \rightarrow C$ be a retraction, i.e. $\gamma$ is a continuous mapping such that $\gamma(x)=x, x \in C$. Let $\Psi:=P+J Q N+K_{P}(I-Q) N$ and $\Psi_{\gamma}:=\Psi \circ \gamma$.

Theorem 2.1 ([12]) Let $C$ be a cone in $X$ and $\Omega_{1}, \Omega_{2}$ be open bounded subsets of $X$ with $\bar{\Omega}_{1} \subset \Omega_{2}$ and $C \cap\left(\bar{\Omega}_{2} \backslash \Omega_{1}\right) \neq \emptyset$. Assume that $L: \operatorname{dom} L \subset X \rightarrow Y$ is a Fredholm operator of index zero and the following conditions are satisfied.

(C1) $Q N: X \rightarrow Y$ is continuous and bounded and $K_{P}(I-Q) N: X \rightarrow X$ is compact on every bounded subset of $X$;

(C2) $L x \neq \lambda N x$ for all $x \in C \cap \partial \Omega_{2} \cap \operatorname{dom} L$ and $\lambda \in(0,1)$;

(C3) $\gamma$ maps subsets of $\bar{\Omega}_{2}$ into bounded subsets of $C$;

(C4) $d_{B}\left(\left.[I-(P+J Q N) \gamma]\right|_{\mathrm{Ker} L}, \operatorname{Ker} L \cap \Omega_{2}, 0\right) \neq 0$, where $d_{B}$ stands for the Brouwer degree;

(C5) there exists $u_{0} \in C \backslash\{0\}$ such that $\|x\| \leq \sigma\left(u_{0}\right)\|\Psi x\|$ for $x \in C\left(u_{0}\right) \cap \partial \Omega_{1}$, where $C\left(u_{0}\right)=\left\{x \in C: \mu u_{0} \preceq x\right.$ for some $\left.\mu>0\right\}$ and $\sigma\left(u_{0}\right)$ is such that $\left\|x+u_{0}\right\| \geq \sigma\left(u_{0}\right)\|x\|$ for every $x \in C ;$

(C6) $(P+J Q N) \gamma\left(\partial \Omega_{2}\right) \subset C$;

(C7) $\Psi_{\gamma}\left(\bar{\Omega}_{2} \backslash \Omega_{1}\right) \subset C$.

Then the equation $L x=N x$ has a solution in the set $C \cap\left(\bar{\Omega}_{2} \backslash \Omega_{1}\right)$.

Lemma 2.1 ([23]) Assume that $V \subset X$ is bounded. $V$ is compact if $\left\{\frac{u(t)}{1+t}: u \in V\right\}$ is equicontinuous on $[0, T], \forall T<\infty$, and equiconvergent at infinity.

In this paper, we will always suppose that the following condition holds.

$\left(\mathrm{A}_{1}\right) f:[0,+\infty) \times \mathbf{R} \rightarrow \mathbf{R}$ is continuous and $f(t, 0)$ is not always equal to 0 . For any $r>0$, there exists $h_{r}(t) \in L[0,+\infty), h_{r}(t)>0$ satisfying $|f(t,(1+t) u)| \leq h_{r}(t), t \in[0,+\infty),|u| \leq r$, $\alpha_{i}>0, \sum_{i=1}^{m-1} \alpha_{i}=1$.

\section{Main result}

Let

$$
X=\left\{u: u \in C[0,+\infty), u(0)=0, \sup _{t \in[0,+\infty)} \frac{|u(t)|}{1+t}<\infty\right\}
$$

with the norm $\|u\|=\sup _{t \in[0,+\infty)} \frac{|u(t)|}{1+t}$, and

$$
Y=\left\{y: y \in C[0,+\infty) \cap L[0,+\infty), \sup _{t \in[0,+\infty)}|y(t)|<+\infty\right\},
$$

with the norm $\|y\|_{1}=\int_{0}^{+\infty}|y(t)| d t+\sup _{t \in[0,+\infty)}|y(t)|$.

It is easy to prove that $(X,\|\cdot\|)$ and $\left(Y,\|\cdot\|_{1}\right)$ are Banach spaces.

Define $L: \operatorname{dom} L \subset X \rightarrow Y$ and $N: X \rightarrow Y$ as follows:

$$
(L u)(t)=-u^{\prime \prime}(t), \quad(N u)(t)=f(t, u(t)), \quad u(t) \in X, t \in[0,+\infty),
$$


where

$$
\operatorname{dom} L=\left\{u(t) \in X \mid u^{\prime \prime}(t) \in Y, u^{\prime}(+\infty)=\sum_{i=1}^{m-1} \alpha_{i} u^{\prime}\left(\xi_{i}\right)\right\} .
$$

Then the boundary value problem (1.1) can be written

$$
(L u)(t)=(N u)(t), \quad u(t) \in \operatorname{dom} L .
$$

For convenience, denote the function $G(t, s)$ as follows:

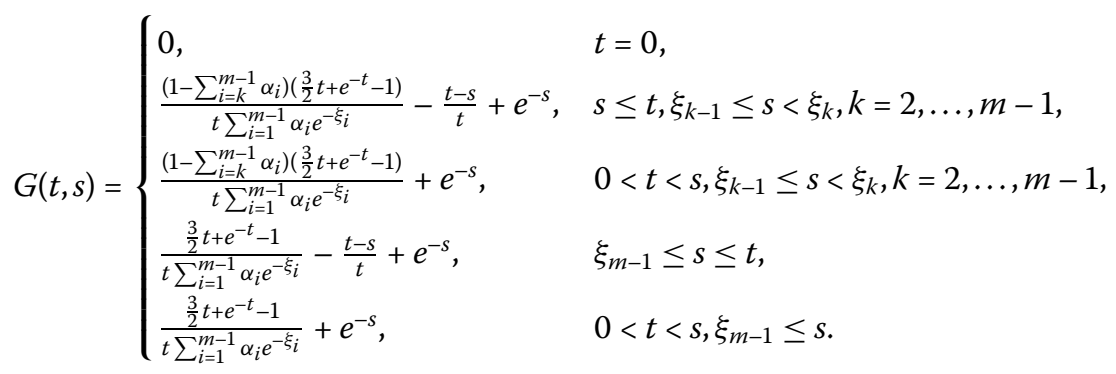

Clearly, $G(t, s) \leq \frac{3}{2 \sum_{i=1}^{m-1} \alpha_{i} e^{-\xi_{i}}}+1$, for $t, s \in[0,+\infty)$.

Lemma 3.1 $L$ is a Fredholm operator of index zero.

Proof It is easy to get

$$
\operatorname{Ker} L=\{u \in \operatorname{dom} L \mid u(t)=c t, t \geq 0, c \in \mathbf{R}\},
$$

and

$$
\operatorname{Im} L=\left\{y \in Y \mid \sum_{i=1}^{m-1} \alpha_{i} \int_{\xi_{i}}^{+\infty} y(s) d s=0\right\} .
$$

Define $Q: Y \rightarrow Y$ by

$$
(Q y)(t)=\frac{e^{-t}}{\sum_{i=1}^{m-1} \alpha_{i} e^{-\xi_{i}}} \sum_{i=1}^{m-1} \alpha_{i} \int_{\xi_{i}}^{+\infty} y(s) d s, \quad y \in Y .
$$

Clearly, $\operatorname{Ker} Q=\operatorname{Im} L, \operatorname{Im} Q=\left\{y \mid y=c e^{-t}, t \geq 0, c \in \mathbf{R}\right\}$, and $Q: Y \rightarrow Y$ is a linear projector. In fact, for $y(t) \in Y$, we have

$$
\left(Q^{2} y\right)(t)=(Q(Q y))(t)=Q\left(e^{-t}\right) \frac{1}{\sum_{i=1}^{m-1} \alpha_{i} e^{-\xi_{i}}} \sum_{i=1}^{m-1} \alpha_{i} \int_{\xi_{i}}^{+\infty} y(s) d s=(Q y)(t) .
$$

For $y \in Y$, we have $y=(y-Q y)+Q y, Q y \in \operatorname{Im} Q,(I-Q) y \in \operatorname{Ker} Q=\operatorname{Im} L$. So we obtain $Y=\operatorname{Im} Q+\operatorname{Im} L$. Take $y_{0} \in \operatorname{Im} Q \cap \operatorname{Im} L . y_{0} \in \operatorname{Im} Q$ means that $y_{0}$ can be written $y_{0}=c e^{-t}$, $c \in \mathbf{R}$. At the same time, by $y_{0} \in \operatorname{Im} L$ and (3.2), we get

$$
\sum_{i=1}^{m-1} \alpha_{i} \int_{\xi_{i}}^{+\infty} c e^{-s} d s=0
$$


i.e. $c=0$. This implies that $y_{0}=0$. Thus, $Y=\operatorname{Im} Q \oplus \operatorname{Im} L$ and $\operatorname{dim} \operatorname{Ker} L=\operatorname{codim} \operatorname{Im} L=1<$ $+\infty$. Observing that $\operatorname{Im} L$ is closed in $Y, L$ is a Fredholm operator of index zero.

Define $P: X \rightarrow X$ as

$$
(P u)(t)=t \int_{0}^{+\infty} e^{-t} u(t) d t, \quad u(t) \in X
$$

Clearly, $P: X \rightarrow X$ is a linear continuous projector and

$$
\operatorname{Im} P=\{u \mid u(t)=c t, t \geq 0, c \in \mathbf{R}\}=\operatorname{Ker} L
$$

Thus, $X=\operatorname{Im} P \oplus \operatorname{Ker} P=\operatorname{Ker} L \oplus \operatorname{Ker} P$.

Define $K_{P}: \operatorname{Im} L \rightarrow \operatorname{Ker} P \cap \operatorname{dom} L$ by

$$
\left(K_{P} y\right)(t)=-\int_{0}^{t}(t-s) y(s) d s+t \int_{0}^{+\infty} e^{-s} y(s) d s, \quad y \in \operatorname{Im} L
$$

By simple calculations, we have $\left(K_{P} L_{P}\right) u=u, \forall u \in \operatorname{dom} L \cap \operatorname{Ker} P$, and $\left(L_{P} K_{P}\right) y=y, \forall y \in$ $\operatorname{Im} L$. So $K_{P}=\left(L_{P}\right)^{-1}$, where $L_{P}=\left.L\right|_{\operatorname{dom} L \cap \operatorname{Ker} P}: \operatorname{dom} L \cap \operatorname{Ker} P \rightarrow \operatorname{Im} L$.

Define the linear isomorphism $J: \operatorname{Im} Q \rightarrow \operatorname{Ker} L$ as

$$
J\left(c e^{-t}\right)=c t, \quad t \geq 0, c \in \mathbf{R} .
$$

Thus, $J Q N+K_{P}(I-Q) N: X \rightarrow X$ is given by

$$
\left[J Q N+K_{P}(I-Q) N\right] u(t)=t \int_{0}^{+\infty} G(t, s) f(s, u(s)) d s .
$$

Lemma 3.2 $Q N: X \rightarrow Y$ is continuous and bounded and $K_{P}(I-Q) N: \bar{\Omega} \rightarrow X$ is compact, where $\Omega \subset X$ is bounded.

Proof For convenience, denote $M_{r}:=\frac{\int_{0}^{+\infty} h_{r}(\tau) d \tau}{\sum_{i=1}^{m-1} \alpha_{i} e^{-\xi_{i}}}$.

We will prove that $Q N: X \rightarrow Y$ is continuous and bounded.

Since $\Omega \subset X$ is bounded, for $u \in \bar{\Omega}$, there exists a constant $r>0$, such that $\|u\|<r$. By the condition $\left(\mathrm{A}_{1}\right)$, we have

$$
\begin{aligned}
& \|Q N u\|_{1}=\int_{0}^{+\infty}\left|\frac{e^{-s}}{\sum_{i=1}^{m-1} \alpha_{i} e^{-\xi_{i}}} \sum_{i=1}^{m-1} \alpha_{i} \int_{\xi_{i}}^{+\infty} f(\tau, u(\tau)) d \tau\right| d s \\
& +\sup _{t \in[0,+\infty)}\left|\frac{e^{-t}}{\sum_{i=1}^{m-1} \alpha_{i} e^{-\xi_{i}}} \sum_{i=1}^{m-1} \alpha_{i} \int_{\xi_{i}}^{+\infty} f(\tau, u(\tau)) d \tau\right| \\
& \leq \int_{0}^{+\infty} \frac{e^{-s}}{\sum_{i=1}^{m-1} \alpha_{i} e^{-\xi_{i}}} \sum_{i=1}^{m-1} \alpha_{i} \int_{\xi_{i}}^{+\infty}|f(\tau, u(\tau))| d \tau d s \\
& +\sup _{t \in[0,+\infty)} \frac{e^{-t}}{\sum_{i=1}^{m-1} \alpha_{i} e^{-\xi_{i}}} \sum_{i=1}^{m-1} \alpha_{i} \int_{\xi_{i}}^{+\infty}|f(\tau, u(\tau))| d \tau \\
& \leq \frac{\int_{0}^{+\infty} h_{r}(\tau) d \tau}{\sum_{i=1}^{m-1} \alpha_{i} e^{-\xi_{i}}}+\frac{\int_{0}^{+\infty} h_{r}(\tau) d \tau}{\sum_{i=1}^{m-1} \alpha_{i} e^{-\xi_{i}}}=2 M_{r}
\end{aligned}
$$


So $Q N: X \rightarrow Y$ is bounded. By $\left(\mathrm{A}_{1}\right)$ and the Lebesgue dominated convergence theorem, we see that $Q N: X \rightarrow Y$ is continuous.

Now, we will prove that $K_{P}(I-Q) N: \bar{\Omega} \rightarrow X$ is compact.

First of all, by the condition $\left(\mathrm{A}_{1}\right)$ and $u \in \bar{\Omega}$, we have

$$
\begin{aligned}
& \left|\frac{K_{P}(I-Q) N u(t)}{1+t}\right| \\
& \quad=\left|-\int_{0}^{t} \frac{t-s}{1+t}(I-Q) N u(s) d s+\frac{t}{1+t} \int_{0}^{+\infty} e^{-s}(I-Q) N u(s) d s\right| \\
& \quad \leq \int_{0}^{t}|(I-Q) N u(s)| d s+\int_{0}^{+\infty}|(I-Q) N u(s)| d s \\
& \quad \leq 2 \int_{0}^{+\infty}|N u(s)| d s+2 \int_{0}^{+\infty}|Q N u(s)| d s \\
& \quad \leq 2 \int_{0}^{\infty} h_{r}(s) d s+2 M_{r} \leq 4 M_{r}<+\infty,
\end{aligned}
$$

i.e. $K_{P}(I-Q) N: \bar{\Omega} \rightarrow X$ is bounded.

Second, for $u \in \bar{\Omega}, 0<t_{1}<t_{2}<T<\infty$,

$$
\begin{aligned}
&\left|\frac{K_{P}(I-Q) N u\left(t_{2}\right)}{1+t_{2}}-\frac{K_{P}(I-Q) N u\left(t_{1}\right)}{1+t_{1}}\right| \\
&=\mid-\int_{0}^{t_{2}} \frac{t_{2}-s}{1+t_{2}}(I-Q) N u(s) d s+\frac{t_{2}}{1+t_{2}} \int_{0}^{+\infty} e^{-s}(I-Q) N u(s) d s \\
& \quad-\left(-\int_{0}^{t_{1}} \frac{t_{1}-s}{1+t_{1}}(I-Q) N u(s) d s+\frac{t_{1}}{1+t_{1}} \int_{0}^{+\infty} e^{-s}(I-Q) N u(s) d s\right) \mid \\
& \leq \int_{0}^{t_{1}}\left|\frac{t_{2}-s}{1+t_{2}}-\frac{t_{1}-s}{1+t_{1}}\right||(I-Q) N u(s)| d s+\int_{t_{1}}^{t_{2}} \frac{t_{2}-s}{1+t_{2}}|(I-Q) N u(s)| d s \\
& \quad+\left|\frac{t_{2}}{1+t_{2}}-\frac{t_{1}}{1+t_{1}}\right| \int_{0}^{+\infty} e^{-s}|(I-Q) N u(s)| d s \\
& \leq \int_{0}^{t_{1}}\left|\frac{t_{2}-s}{1+t_{2}}-\frac{t_{1}-s}{1+t_{1}}\right| h_{r}(s) d s+\int_{0}^{t_{1}}\left|\frac{t_{2}-s}{1+t_{2}}-\frac{t_{1}-s}{1+t_{1}}\right||Q N u(s)| d s \\
& \quad+\int_{t_{1}}^{t_{2}} \frac{t_{2}-s}{1+t_{2}} h_{r}(s) d s+\int_{t_{1}}^{t_{2}} \frac{t_{2}-s}{1+t_{2}}|Q N u(s)| d s \\
& \quad+\left|\frac{t_{2}}{1+t_{2}}-\frac{t_{1}}{1+t_{1}}\right| \int_{0}^{+\infty} h_{r}(s) d s+\left|\frac{t_{2}}{1+t_{2}}-\frac{t_{1}}{1+t_{1}}\right| \int_{0}^{+\infty}|Q N u(s)| d s \\
& \leq \int_{0}^{t_{1}}\left|\frac{t_{2}-s}{1+t_{2}}-\frac{t_{1}-s}{1+t_{1}}\right| h_{r}(s) d s+M_{r} \int_{0}^{t_{1}}\left|\frac{t_{2}-s}{1+t_{2}}-\frac{t_{1}-s}{1+t_{1}}\right| e^{-s} d s \\
&+\int_{t_{1}}^{t_{2}} h_{r}(s) d s+M_{r} \int_{t_{1}}^{t_{2}} e^{-s} d s+2\left|\frac{t_{2}}{1+t_{2}}-\frac{t_{1}}{1+t_{1}}\right| M_{r} .
\end{aligned}
$$

By the uniform continuity of $\frac{t-s}{1+t}$ in $[0, T] \times[0, T]$ and $\frac{t}{1+t}$ in $[0, T]$, and the absolute continuity of the integral, we see that $K_{P}(I-Q) N: \bar{\Omega} \rightarrow X$ is equicontinuous on [0,T], $\forall T>0$. 
Third, for $\varepsilon>0$, there exists a constant $l>0$, such that

$$
\int_{l}^{+\infty} h_{r}(s) d s<\frac{\varepsilon}{12}, \quad \int_{l}^{+\infty} e^{-s} d s<\frac{\varepsilon}{12 M_{r}}
$$

Since $\lim _{t \rightarrow+\infty} \frac{t-l}{1+t}=1, \lim _{t \rightarrow+\infty} \frac{t}{1+t}=1$, there exists a constant $T>l$ such that

$$
\left|1-\frac{t-l}{1+t}\right|<\frac{\varepsilon}{12 M_{r}}, \quad\left|1-\frac{t}{1+t}\right|<\frac{\varepsilon}{12 M_{r}}, \quad t \geq T .
$$

For $u \in \bar{\Omega}, T \leq t_{1}<t_{2}$, we have

$$
\begin{aligned}
& \left|\frac{K_{P}(I-Q) N u\left(t_{2}\right)}{1+t_{2}}-\frac{K_{P}(I-Q) N u\left(t_{1}\right)}{1+t_{1}}\right| \\
& =\mid-\int_{0}^{t_{2}} \frac{t_{2}-s}{1+t_{2}}(I-Q) N u(s) d s+\frac{t_{2}}{1+t_{2}} \int_{0}^{+\infty} e^{-s}(I-Q) N u(s) d s \\
& -\left(-\int_{0}^{t_{1}} \frac{t_{1}-s}{1+t_{1}}(I-Q) N u(s) d s+\frac{t_{1}}{1+t_{1}} \int_{0}^{+\infty} e^{-s}(I-Q) N u(s) d s\right) \\
& \leq \int_{0}^{l}\left|\frac{t_{2}-s}{1+t_{2}}-\frac{t_{1}-s}{1+t_{1}}\right||(I-Q) N u(s)| d s+\int_{l}^{t_{2}} \frac{t_{2}-s}{1+t_{2}}|(I-Q) N u(s)| d s \\
& +\int_{l}^{t_{1}} \frac{t_{1}-s}{1+t_{1}}|(I-Q) N u(s)| d s+\left|\frac{t_{2}}{1+t_{2}}-\frac{t_{1}}{1+t_{1}}\right| \int_{0}^{+\infty} e^{-s}|(I-Q) N u(s)| d s \\
& \leq\left[\left(1-\frac{t_{2}-l}{1+t_{2}}\right)+\left(1-\frac{t_{1}-l}{1+t_{1}}\right)\right] \int_{0}^{+\infty}|(I-Q) N u(s)| d s+2 \int_{l}^{+\infty}|(I-Q) N u(s)| d s \\
& +\left[\left(1-\frac{t_{2}}{1+t_{2}}\right)+\left(1-\frac{t_{1}}{1+t_{1}}\right)\right] \int_{0}^{+\infty}|(I-Q) N u(s)| d s \\
& \leq\left[\left(1-\frac{t_{2}-l}{1+t_{2}}\right)+\left(1-\frac{t_{1}-l}{1+t_{1}}\right)\right] 2 M_{r}+2 \int_{l}^{+\infty} h_{r}(s) d s+2 M_{r} \int_{l}^{+\infty} e^{-s} d s \\
& +\left[\left(1-\frac{t_{2}}{1+t_{2}}\right)+\left(1-\frac{t_{1}}{1+t_{1}}\right)\right] 2 M_{r}<\varepsilon \text {. }
\end{aligned}
$$

Thus, $K_{P}(I-Q) N: \bar{\Omega} \rightarrow X$ is equiconvergent at infinity.

By Lemma 2.1, we see that $K_{P}(I-Q) N: \bar{\Omega} \rightarrow X$ is compact.

Theorem 3.1 Assume that $\left(\mathrm{A}_{1}\right)$ and the following conditions hold.

$\left(\mathrm{A}_{2}\right)$ For $u \geq 0$, there exist three nonnegative functions $\mu(t), \beta_{i}(t), i=1,2$, such that

$$
-\mu(t) u e^{-t} \leq f(t, u) \leq-\beta_{1}(t) u e^{-t}+\beta_{2}(t), \quad G(t, s) f(s, u) \geq-e^{-s} u, \quad t, s \in[0,+\infty),
$$

where $\mu(t) u e^{-t}, \beta_{2}(t), \beta_{1}(t) u e^{-t} \in L[0,+\infty)$ inf $_{t \in[0,+\infty)} \beta_{1}(t):=\beta_{0}>0$ and $\mu(t)$ satisfying

(i) $\sup _{t \in[0,+\infty)} \mu(t):=\mu_{1}<\frac{2 \sum_{i=1}^{m-1} \alpha_{i} e^{-\xi_{i}}}{3+2 \sum_{i=1}^{m-1} \alpha_{i} e^{-\xi_{i}}}$,

(ii) there exists $t_{0} \in[0,+\infty)$, such that $d_{0}:=\frac{t_{0}}{1+t_{0}} \int_{0}^{+\infty}\left[1-G\left(t_{0}, s\right) \mu(s)\right](1+s) e^{-s} d s>1$, $G\left(t_{0}, s\right) \geq 0$.

$\left(\mathrm{A}_{3}\right)$ There exists $R>\frac{\mu_{1}+\alpha_{1} \beta_{0}+1}{\alpha_{1} \beta_{0}} \int_{0}^{\infty} \beta_{2}(s) d s$, such that $f(t, R t)<0, t \in[0,+\infty)$.

Then the problem (1.1) has at least one positive solution. 
Proof Take a cone

$$
C=\{u(t) \in X \mid u(t) \geq 0, t \in[0,+\infty)\}
$$

Set

$$
\Omega_{1}=\left\{u \in X \mid \frac{1}{d_{0}}\|u\|<\frac{|u(t)|}{1+t}<r<R, t \in[0,+\infty)\right\}, \quad \Omega_{2}=\{u \in X \mid\|u\|<R\},
$$

where $d_{0}$ is given by the condition $\left(\mathrm{A}_{2}\right)$ and $R>\frac{\mu_{1}+\alpha_{1} \beta_{0}+1}{\alpha_{1} \beta_{0}} \int_{0}^{\infty} \beta_{2}(s) d s$. Clearly, $\Omega_{1}$, $\Omega_{2}$ are open and bounded sets of $X, \bar{\Omega}_{1}=\left\{u \in X \mid \frac{1}{d_{0}}\|u\| \leq \frac{|u(t)|}{1+t} \leq r<R\right\} \subset \Omega_{2}$, and $C \cap\left(\bar{\Omega}_{2} \backslash \Omega_{1}\right) \neq \emptyset$.

In view of Lemmas 3.1 and 3.2, $L$ is a Fredholm operator of index zero and the condition (C1) of Theorem 2.1 is fulfilled.

Suppose that there exist $u_{1}(t) \in C \cap \partial \Omega_{2} \cap \operatorname{dom} L$ and $\lambda_{0} \in(0,1)$ such that $L u_{1}=\lambda_{0} N u_{1}$, i.e. $u_{1}^{\prime \prime}(t)+\lambda_{0} f\left(t, u_{1}(t)\right)=0$. By $u_{1}(t) \in \operatorname{dom} L$, we have

$$
\begin{aligned}
& u_{1}^{\prime}(+\infty)-\sum_{i=1}^{m-1} \alpha_{i} u_{1}^{\prime}\left(\xi_{i}\right)=0, \\
& \text { i.e. }-\lambda_{0} \int_{0}^{+\infty} f\left(s, u_{1}(s)\right) d s+\sum_{i=1}^{m-1} \alpha_{i} \lambda_{0} \int_{0}^{\xi_{i}} f\left(s, u_{1}(s)\right) d s=0 .
\end{aligned}
$$

It follows from $\left(\mathrm{A}_{2}\right)$ that

$$
0=\sum_{i=1}^{m-1} \alpha_{i} \int_{\xi_{i}}^{+\infty} f\left(s, u_{1}(s)\right) d s \leq \sum_{i=1}^{m-1} \alpha_{i} \int_{\xi_{i}}^{+\infty}\left[-\beta_{1}(s) u_{1}(s) e^{-s}+\beta_{2}(s)\right] d s .
$$

So

$$
\alpha_{1} \int_{0}^{+\infty} \beta_{1}(s) u_{1}(s) e^{-s} d s \leq \int_{0}^{+\infty} \beta_{2}(s) d s
$$

Considering $\left(\mathrm{A}_{2}\right)$, (3.7), and

$$
\begin{aligned}
u_{1}(t) & =(I-P) u_{1}(t)+P u_{1}(t)=K_{P} L(I-P) u_{1}(t)+P u_{1}(t)=K_{P} L u_{1}(t)+P u_{1}(t) \\
& =-\lambda_{0} \int_{0}^{t}(t-s) f\left(s, u_{1}(s)\right) d s+\lambda_{0} t \int_{0}^{+\infty} e^{-s} f\left(s, u_{1}(s)\right) d s+t \int_{0}^{+\infty} e^{-s} u_{1}(s) d s,
\end{aligned}
$$

we obtain

$$
\begin{aligned}
\frac{u_{1}(t)}{1+t}= & -\frac{\lambda_{0}}{1+t} \int_{0}^{t}(t-s) f\left(s, u_{1}(s)\right) d s \\
& +\frac{\lambda_{0} t}{1+t} \int_{0}^{+\infty} e^{-s} f\left(s, u_{1}(s)\right) d s+\frac{t}{1+t} \int_{0}^{+\infty} e^{-s} u_{1}(s) d s \\
\leq & \lambda_{0} \int_{0}^{t} \frac{(t-s)}{1+t} \mu(s) u_{1}(s) e^{-s} d s \\
& +\frac{\lambda_{0} t}{1+t} \int_{0}^{+\infty} e^{-s}\left[-\beta_{1}(s) u_{1}(s) e^{-s}+\beta_{2}(s)\right] d s+\frac{t}{1+t} \int_{0}^{+\infty} e^{-s} u_{1}(s) d s
\end{aligned}
$$




$$
\begin{aligned}
\leq & \int_{0}^{t} \frac{\beta_{1}(s) e^{-s} \mu(s) u_{1}(s)}{\beta_{1}(s)} d s+\int_{0}^{+\infty} \beta_{2}(s) d s+\int_{0}^{+\infty} \frac{\beta_{1}(s) e^{-s} u_{1}(s)}{\beta_{1}(s)} d s \\
\leq & \frac{\mu_{1}+\alpha_{1} \beta_{0}+1}{\alpha_{1} \beta_{0}} \int_{0}^{+\infty} \beta_{2}(s) d s<R, \\
\frac{u_{1}(t)}{1+t}= & -\frac{\lambda_{0}}{1+t} \int_{0}^{t}(t-s) f\left(s, u_{1}(s)\right) d s \\
& +\frac{\lambda_{0} t}{1+t} \int_{0}^{+\infty} e^{-s} f\left(s, u_{1}(s)\right) d s+\frac{t}{1+t} \int_{0}^{+\infty} e^{-s} u_{1}(s) d s \\
\geq & \lambda_{0} \int_{0}^{t} \frac{t-s}{1+t}\left[\beta_{1}(s) u_{1}(s) e^{-s}-\beta_{2}(s)\right] d s \\
& +\frac{\lambda_{0} t}{1+t} \int_{0}^{+\infty} e^{-s}\left[-\mu(s) u_{1}(s) e^{-s}\right] d s+\frac{t}{1+t} \int_{0}^{+\infty} e^{-s} u_{1}(s) d s \\
\geq & -\int_{0}^{t} \beta_{2}(s) d s-\int_{0}^{+\infty} \frac{\beta_{1}(s) \mu(s) e^{-s} u_{1}(s)}{\beta_{1}(s)} d s \\
\geq & -\frac{\alpha_{1} \beta_{0}+\mu_{1}}{\alpha_{1} \beta_{0}} \int_{0}^{+\infty} \beta_{2}(s) d s>-R .
\end{aligned}
$$

These contradict $u_{1}(t) \in C \cap \partial \Omega_{2} \cap \operatorname{dom} L$. So (C2) is satisfied.

Let $(\gamma u)(t)=|u(t)|, u(t) \in X$. Then $\gamma: X \rightarrow C$ is a retraction and maps subsets of $\bar{\Omega}_{2}$ into bounded subsets of $C$, i.e. (C3) holds.

Let $u(t) \in \operatorname{Ker} L \cap \partial \Omega_{2}$, then $u(t)=c t, t \geq 0$. Define

$$
\begin{aligned}
H(c t, \lambda) & =[I-\lambda(P+J Q N) \gamma](c t) \\
& =c t-\lambda t \int_{0}^{+\infty} e^{-t}|c| t d t-\frac{\lambda t}{\sum_{i=1}^{m-1} \alpha_{i} e^{-\xi_{i}}} \sum_{i=1}^{m-1} \alpha_{i} \int_{\xi_{i}}^{+\infty} f(t,|c| t) d t,
\end{aligned}
$$

where $c \in\{-R, R\}$ and $\lambda \in[0,1]$. Suppose $H(c t, \lambda)=0$, by $\left(\mathrm{A}_{2}\right)$, we obtain

$$
\begin{aligned}
c & =\lambda\left(|c|+\frac{1}{\sum_{i=1}^{m-1} \alpha_{i} e^{-\xi_{i}}} \sum_{i=1}^{m-1} \alpha_{i} \int_{\xi_{i}}^{+\infty} f(t,|c| t) d t\right) \\
& \geq \lambda|c|\left(1-\frac{1}{\sum_{i=1}^{m-1} \alpha_{i} e^{-\xi_{i}}} \sum_{i=1}^{m-1} \alpha_{i} \int_{\xi_{i}}^{+\infty} \mu(t) t e^{-t} d t\right) \\
& \geq \lambda|c|\left(1-\frac{\mu_{1}}{\sum_{i=1}^{m-1} \alpha_{i} e^{-\xi_{i}}} \sum_{i=1}^{m-1} \alpha_{i} \int_{0}^{+\infty} t e^{-t} d t\right) \\
& =\lambda|c|\left(1-\frac{\mu_{1}}{\sum_{i=1}^{m-1} \alpha_{i} e^{-\xi_{i}}}\right) \geq 0 .
\end{aligned}
$$

Hence $H(c t, \lambda)=0$ implies $c \geq 0$. Furthermore, if $H(R t, \lambda)=0$, we have

$$
R(1-\lambda)=\frac{\lambda}{\sum_{i=1}^{m-1} \alpha_{i} e^{-\xi_{i}}} \sum_{i=1}^{m-1} \alpha_{i} \int_{\xi_{i}}^{+\infty} f(t, R t) d t \geq 0,
$$

which is a contradiction to the condition $\left(\mathrm{A}_{3}\right)$. 
Thus, $H(u, \lambda) \neq 0$, for $u \in \operatorname{Ker} L \cap \partial \Omega_{2}$, and $\lambda \in[0,1]$. Therefore

$$
\begin{aligned}
& d_{B}\left(\left.[I-(P+J Q N) \gamma]\right|_{\operatorname{Ker} L}, \operatorname{Ker} L \cap \Omega_{2}, 0\right) \\
& \quad=d_{B}\left(H(\cdot, 1), \operatorname{Ker} L \cap \Omega_{2}, 0\right) \\
& \quad=d_{B}\left(H(\cdot, 0), \operatorname{Ker} L \cap \Omega_{2}, 0\right)=d_{B}\left(I, \operatorname{Ker} L \cap \Omega_{2}, 0\right)=1 \neq 0 .
\end{aligned}
$$

Thus, (C4) holds.

Let $u_{0}(t)=t, t \in[0,+\infty)$, then $u_{0} \in C \backslash\{0\}, C\left(u_{0}\right)=\{u \in C \mid u(t) \geq \mu t$ for some $\mu>$ $0, t \in[0,+\infty)\}$, and we take $\sigma\left(u_{0}\right)=1$. Let $u \in C\left(u_{0}\right) \cap \partial \Omega_{1}$, we have $\frac{1}{d_{0}}\|u\| \leq \frac{|u(t)|}{1+t} \leq r$, $t \in[0,+\infty)$.

For $u \in C\left(u_{0}\right) \cap \partial \Omega_{1}$, by $\left(\mathrm{A}_{2}\right)$, we get

$$
\begin{aligned}
\frac{\Psi u\left(t_{0}\right)}{1+t_{0}} & =\frac{t_{0}}{1+t_{0}} \int_{0}^{+\infty} e^{-s} u(s) d s+\frac{t_{0}}{1+t_{0}} \int_{0}^{+\infty} G\left(t_{0}, s\right) f(s, u(s)) d s \\
& \geq \frac{t_{0}}{1+t_{0}} \int_{0}^{+\infty}\left(e^{-s} u(s)-G\left(t_{0}, s\right) \mu(s) u(s) e^{-s}\right) d s \\
& =\frac{t_{0}}{1+t_{0}} \int_{0}^{+\infty}\left[1-G\left(t_{0}, s\right) \mu(s)\right](1+s) e^{-s} \frac{u(s)}{1+s} d s \\
& \geq \frac{t_{0}}{1+t_{0}} \int_{0}^{+\infty}\left[1-G\left(t_{0}, s\right) \mu(s)\right](1+s) e^{-s} d s \frac{1}{d_{0}}\|u\|=\|u\| .
\end{aligned}
$$

Thus, $\|u\| \leq \sigma\left(u_{0}\right)\|\Psi u\|$, for $u \in C\left(u_{0}\right) \cap \partial \Omega_{1}$. So (C5) holds.

For $u(t) \in \partial \Omega_{2}, t \in[0,+\infty)$, by the condition $\left(\mathrm{A}_{2}\right)$, we have

$$
\begin{aligned}
(P+J Q N) \gamma(u) & =t \int_{0}^{+\infty} e^{-s}|u(s)| d s+\frac{t}{\sum_{i=1}^{m-1} \alpha_{i} e^{-\xi_{i}}} \sum_{i=1}^{m-1} \alpha_{i} \int_{\xi_{i}}^{+\infty} f(s,|u(s)|) d s \\
& \geq t \int_{0}^{+\infty} e^{-s}|u(s)| d s-\frac{t}{\sum_{i=1}^{m-1} \alpha_{i} e^{-\xi_{i}}} \sum_{i=1}^{m-1} \alpha_{i} \int_{\xi_{i}}^{+\infty} \mu(s)|u(s)| e^{-s} d s \\
& \geq t \int_{0}^{+\infty} e^{-s}|u(s)| d s-\frac{t}{\sum_{i=1}^{m-1} \alpha_{i} e^{-\xi_{i}}} \int_{0}^{+\infty} \mu(s)|u(s)| e^{-s} d s \\
& =t \int_{0}^{+\infty} e^{-s}|u(s)|\left(1-\frac{\mu(s)}{\sum_{i=1}^{m-1} \alpha_{i} e^{-\xi_{i}}}\right) d s \geq 0,
\end{aligned}
$$

which means that $(P+J Q N) \gamma\left(\partial \Omega_{2}\right) \subset C$. Hence, $($ C6 $)$ holds.

For $u(t) \in \bar{\Omega}_{2} \backslash \Omega_{1}, t \in[0,+\infty)$, by the condition $\left(\mathrm{A}_{2}\right)$, we have

$$
\begin{aligned}
\left(\Psi_{\gamma} u\right)(t) & =t \int_{0}^{+\infty} e^{-s}|u(s)| d s+t \int_{0}^{+\infty} G(t, s) f(s,|u(s)|) d s \\
& \geq t \int_{0}^{+\infty} e^{-s}|u(s)| d s-t \int_{0}^{+\infty} e^{-s}|u(s)| d s \\
& =0 .
\end{aligned}
$$

So $\Psi_{\gamma}\left(\bar{\Omega}_{2} \backslash \Omega_{1}\right) \subset C$, i.e. $(\mathrm{C} 7)$ is satisfied.

By Theorem 2.1, we confirm that the equation $L u=N u$ has a positive solution $u$, i.e. the problem (1.1) has at least one positive solution. 


\section{Examples}

Let us consider the following boundary value problem:

$$
\left\{\begin{array}{l}
u^{\prime \prime}(t)+t e^{-t}-\frac{1}{90} u(t) e^{-t}=0, \quad t \in[0,+\infty) \\
u(0)=0, \quad u^{\prime}(+\infty)=0.68 u^{\prime}(0)+0.018 u^{\prime}(0.5)+0.302 u^{\prime}(0.95) .
\end{array}\right.
$$

Here, $f(t, u(t))=t e^{-t}-\frac{1}{90} u(t) e^{-t}, \alpha_{1}=0.68, \alpha_{2}=0.018, \alpha_{3}=0.302, \xi_{1}=0, \xi_{2}=0.5, \xi_{3}=$ 0.95. Take $h_{r}(t)=t e^{-t}+\frac{r}{90}(1+t) e^{-t}, \mu(t)=\frac{1}{80}, \beta_{1}(t)=\frac{1}{100}, \beta_{2}(t)=t e^{-t}, t \in[0,+\infty), t_{0}=1.05$, $R=160, r=150$.

Obviously, $|f(t,(1+t) u)| \leq h_{r}(t), t \in[0,+\infty), r>0,|u|<r$. By our calculations, we can get $0.0029 \leq G(t, s) \leq 2.8571$ and

$$
\begin{aligned}
& -\mu(t) u e^{-t} \leq f(t, u) \leq-\beta_{1}(t) u e^{-t}+\beta_{2}(t), \\
& G(t, s) f(s, u)>-e^{-s} u, \quad u \geq 0, t \in[0,+\infty),
\end{aligned}
$$

$\mu_{1}=\sup _{t \in[0,+\infty)} \mu(t)=\frac{1}{80}, \beta_{0}=\inf _{t \in[0,+\infty)} \beta_{1}(t)=\frac{1}{100}, \beta_{2}(t) \in L[0,+\infty) ; f(t, R t)<0, t \in$ $[0,+\infty)$. By simple calculations, we can get that $0.348<G(1.05, s)<1.478$, so

$$
G(1.05, s)>0, \quad d_{0}:=\frac{1.05}{1+1.05} \int_{0}^{+\infty}[1-G(1.05, s) \mu(s)](1+s) e^{-s} d s \geq 1.005>1
$$

So the conditions $\left(\mathrm{A}_{1}\right)-\left(\mathrm{A}_{3}\right)$ hold. By Theorem 3.1, we can conclude that the problem (4.1) has at least one positive solution.

\section{Competing interests}

The authors declare that there is no conflict of interests regarding the publication of this article.

\section{Authors' contributions}

The authors declare that the study was realized in collaboration with the same responsibility. All authors read and approved the final manuscript.

\section{Acknowledgements}

The authors are grateful to anonymous referees for their constructive comments and suggestions which led to improvement of the original manuscript. This work is supported by the Natural Science Foundation of China (11171088) and the Natural Science Foundation of Hebei Province (A2013208108).

Received: 27 August 2015 Accepted: 30 December 2015 Published online: 13 January 2016

\section{References}

1. Gupta, CP: Existence theorems for a second order $m$-point boundary value problem at resonance. Int. J. Math. Math. Sci. 18, 705-710 (1995)

2. Feng, W, Webb, JRL: Solvability of three-point boundary value problems at resonance. Nonlinear Anal. 30, 3227-3238 (1997)

3. Du, Z, Lin, X, Ge, W: Some higher-order multi-point boundary value problem at resonance. J. Comput. Appl. Math. $177,55-65(2005)$

4. Liu, Y, Shen, C: On the existence of positive solution for a kind of multi-point boundary value problem at resonance. Nonlinear Anal. 72, 4211-4220 (2010)

5. Liu, B: Solvability of multi-point boundary value problem at resonance (II). Appl. Math. Comput. 136, 353-377 (2003)

6. Ma, R: Existence results of a $m$-point boundary value problem at resonance. J. Math. Anal. Appl. 294, 147-157 (2004)

7. Ma, R: Multiplicity results for a three-point boundary value problem at resonance. Nonlinear Anal. 53, 777-789 (2003)

8. Han, X: Positive solutions for a three-point boundary value problem at resonance. J. Math. Anal. Appl. 336, 556-568 (2007)

9. Zhang, X, Feng, M, Ge, W: Existence result of second-order differential equations with integral boundary conditions at resonance. J. Math. Anal. Appl. 353, 311-319 (2009)

10. Jiang, W: Solvability for $p$-Laplacian boundary value problem at resonance on the half-line. Bound. Value Probl. 2013, 207 (2013)

11. Bai, C, Fang, J: Existence of positive solutions for three-point boundary value problems at resonance. J. Math. Anal. Appl. 291, 538-549 (2004) 
12. O'Regan, D, Zima, M: Leggett-Williams norm-type theorems for coincidences. Arch. Math. 87, $233-244$ (2006)

13. Liang, S, Mu, L: Multiplicity of positive solutions for singular three-point boundary value problems at resonance. Nonlinear Anal. 71, 2497-2505 (2009)

14. Zima, M, Drygas, P: Existence of positive solutions for a kind of periodic boundary value problem at resonance. Bound. Value Probl. 2013, 19 (2013)

15. Zhang, H, Sun, J: Positive solutions of third-order nonlocal boundary value problems at resonance. Bound. Value Probl. 2012, 102 (2012)

16. Yang, L, Zhang, W: Positive solution for second order multi-point boundary value problem at resonance. Fixed Point Theory 14, 401-412 (2013)

17. Infante, G, Zima, M: Positive solutions of multi-point boundary value problems at resonance. Nonlinear Anal. 69 , 2458-2465 (2008)

18. Liu, B, Li, J, Liu, L: Existence and uniqueness for an $m$-point boundary value problem at resonance on infinite intervals. Comput. Math. Appl. 64, 1677-1690 (2012)

19. Li, J, Liu, B, Liu, L: Solutions for a boundary value problem at resonance on $[0,+\infty)$. Math. Comput. Model. 58, 1769-1773 (2013)

20. Lian, H, Pang, H, Ge, W: Solvability for second-order three-point boundary value problem at resonance on a half-line. J. Math. Anal. Appl. 337, 1171-1181 (2008)

21. Yang, A, Ge, W: Positive solutions for second-order boundary value problem with integral boundary conditions at resonance on a half-line. JIPAM. J. Inequal. Pure Appl. Math. 10, 9 (2009)

22. Chen, Y, Tang, X: Positive solutions of fractional differential equations at resonance on the half-line. Bound. Value Probl. 2012, 64 (2012)

23. Kosmatov, N: Multi-point boundary value problems on an unbounded domain at resonance. Nonlinear Anal. 68 2158-2171 (2008)

\section{Submit your manuscript to a SpringerOpen ${ }^{\circ}$ journal and benefit from:}

- Convenient online submission

Rigorous peer review

- Immediate publication on acceptance

- Open access: articles freely available online

- High visibility within the field

- Retaining the copyright to your article 